\title{
The effect of ethanol and heat on the functional hydrophobicity of casein micelles
}

\author{
R. Trejo and F. Harte ${ }^{1}$ \\ Department of Food Science and Technology, University of Tennessee, Knoxville 37996-4539
}

\begin{abstract}
Milk proteins are very important ingredients to the food industry. As new uses and applications for these proteins are developed, it becomes more important to understand their physicochemical properties when they are subjected to different treatments. It has been reported that casein micelles dissociate when heated in the presence of ethanol. The changes to the hydrophobicity of milk proteins during that process were evaluated by using the fluorescent hydrophobic probe 1-anilinonaphthalene-8-sulfonic acid (ANS). Raw skim milk, pasteurized skim milk, and whey protein isolate samples with ethanol concentrations of 0 to $60 \%$ ( $\mathrm{vol} /$ vol) were heated from 20 to $60^{\circ} \mathrm{C}$. The fluorescence of the samples with and without the addition of ANS was measured at an excitation wavelength of $390 \mathrm{~nm}$ and an emission wavelength of 400 to $500 \mathrm{~nm}$. The results showed a decrease in the extrinsic fluorescence of the samples as the ethanol concentration and temperature increased, indicating competitive inhibition of the ANShydrophobic site interaction by ethanol. This inhibition was further enhanced by the addition of heat. This resulted in a reduction in the functional hydrophobicity of the milk proteins as ethanol rendered the hydrophobic sites unavailable for interaction.
\end{abstract}

Key words: casein, fluorescence, ethanol, hydrophobicity

\section{INTRODUCTION}

Milk continues to be a fundamental element of the human diet, and the use of milk proteins in the food industry continues to increase as more applications are developed. Hydrophobicity is an important property of proteins, highly related to their stability, conformation, and functionality (Kato and Nakai, 1980). Surface (or effective) hydrophobicity is considered one of the most important properties determining protein functionality (Gatti et al., 1995). The net hydrophobicity of the proteins can vary due to conformational changes

Received November 17, 2009.

Accepted March 3, 2010.

${ }^{1}$ Corresponding author: fede@utk.edu induced by the physicochemical stimuli to which milk is subjected during processing and storage (Bonomi et al., 1988; Bonomi and Iametti, 1991; Gatti et al., 1995). Heat treatment can lead to conformational changes in the proteins, such as denaturation and structural rearrangement, which in turn may increase exposure of hydrophobic amino acid residues, altering the functional properties of the protein (Nakai and Li-Chan, 1998).

It has been reported that the milk casein micelle undergoes a semireversible dissociation when heated to $60^{\circ} \mathrm{C}$ in the presence of ethanol (Dunkerley et al., 1993; Zadow, 1993; O'Connell et al., 2001a). Under these conditions, a naturally opaque skim milk solution containing at least 30\% ethanol (vol/vol) becomes translucent when heated. The change in turbidity is due to the dissociation of the casein micelles into their single casein protein constituents. This change is reversible and the solution will regain its opaque color upon cooling. The objective of this study was to determine the changes in hydrophobicity of the casein micelles during dissociation.

Fluorescent markers have been used effectively to monitor hydrophobicity changes in proteins (Hayakawa and Nakai, 1985; Gatti et al., 1995; Alizadeh-Pasdar and Li-Chan, 2000; Risso et al., 2008). The fluorescent marker 1-anilinonaphthalene-8-sulfonic acid (ANS) is used in hydrophobicity studies because of its affinity toward protein hydrophobic sites (Bonomi et al., 1988). The objective of this study was to use the changes in fluorescence intensity of ANS to effectively track variations in the surface hydrophobicity of casein micelles during heat- and ethanol-induced dissociation.

\section{MATERIALS AND METHODS}

\section{Milk Source and Sample Preparation}

Commercially available pasteurized skim milk was obtained from a local grocery store. Raw milk was obtained from a local dairy farm and skimmed by centrifugation $(6,414 \times g$ for $20 \mathrm{~min})$. Whey protein isolate (WPI), produced by spray-drying concentrated fresh sweet whey (BiPro, Davisco Foods International Inc., Eden Prairie, MN) was used to prepare a solution by mixing the isolate with deionized water (wt/vol) to 
obtain a protein concentration similar to that of whey proteins found in milk $(0.8 \%)$. All samples were stored at $4^{\circ} \mathrm{C}$ and used within $3 \mathrm{~d}$. Samples containing milk, ethanol, and deionized water were prepared using the following procedure: all of the samples contained $4 \%$ milk (vol/vol) for fluorescence studies and 10\% milk ( $\mathrm{vol} / \mathrm{vol}$ ) for absorbance studies, in one of the following solutions: 0, 10, 20, 30, 40, 50, and $60 \%$ (vol/vol) ethanol (99 proof, Fisher Scientific, Fair Lawn, NJ) in deionized water. The temperature of the samples was maintained by using a temperature-controlled water bath (Fisher Scientific). Samples containing raw milk and samples containing WPI were prepared as controls.

\section{Spectrophotometry and Spectrophotofluorometry}

Absorbance values for the samples were measured using a spectrophotometer (Genesys2, Thermo Spectronic, Madison, WI) at a wavelength of $500 \mathrm{~nm}$. The samples were measured at $10^{\circ} \mathrm{C}$ intervals from 20 to $60^{\circ} \mathrm{C}$.

Fluorescence spectroscopy was done following the methodology previously used (Bonomi et al., 1988; Altuner et al., 2006) with modifications. Briefly, 6 stock solutions of ANS (Fisher Scientific) were prepared to ensure a final ANS concentration in the measurement cuvette of $2,4,6,8,10$, and $12 \times 10^{-9} M$ when 50 $\mu \mathrm{L}$ of the stock solution was added to a $2-\mathrm{mL}$ volume of sample. The stock solutions were prepared from an initial 0.1 $M$ ANS solution in deionized water solution. The samples containing ANS were constantly stirred inside the cuvette to ensure proper mixing, and temperature-controlled water was constantly circulated through the cuvette holder to maintain the sample temperature. Fluorescence measurements were done using an RF-1501 spectrophotofluorometer (Shimadzu, Columbia, MD) set at excitation wavelength $\left(\boldsymbol{\lambda}_{\mathbf{E X}}\right)=$ $390 \mathrm{~nm}$ and emission wavelength $\left(\boldsymbol{\lambda}_{\mathrm{EM}}\right)=400-500$ $\mathrm{nm}$. The intrinsic fluorescence intensity values $\left(\mathbf{I}_{\mathbf{I f}}\right)$ of the samples without ANS were measured as controls. These measurements were taken at $10^{\circ} \mathrm{C}$ intervals from 20 to $60^{\circ} \mathrm{C}$. After measuring the intrinsic fluorescence, a set volume of $50 \mu \mathrm{L}$ of stock ANS solution was added to the cuvette and the relative fluorescence intensity value was recorded. This procedure was repeated for all ANS concentrations to obtain a concentration gradient. Separate cuvettes were used for each of the stock solutions. The spectrophotometry and the spectrophotofluorometry experiments were repeated 3 times. The average of the measurements collected was used for all calculations.

The extrinsic fluorescence intensity $\left(\mathbf{E}_{\mathbf{I f}(\mathbf{A N S})}\right)$ was calculated by using the following formula: $\mathrm{E}_{\mathrm{If} \text { (ANS) }}=$ $\mathrm{I}_{\text {If (sample+ANS) }}-\mathrm{I}_{\text {If (sample) }}$. This measured the amount of the fluorescence intensity that was due to the ANS interaction with hydrophobic zones.

\section{Hydrophobicity Calculations}

Hydrophobicity of the milk proteins was calculated based on the method described by Kato and Nakai (1980) with modifications. For $\lambda_{\mathrm{EM}}=390 \mathrm{~nm}$, peak fluorescence was found at $\lambda_{\mathrm{EM}}=\sim 450 \mathrm{~nm}$ for all treatments, and this emission wavelength was used for hydrophobicity calculations. The fluorescence intensity peak of ANS when interacting with hydrophobic zones was recorded at $\lambda_{\mathrm{EM}}=480 \mathrm{~nm}$ (Bonomi et al., 1988). However, those experiments did not involve ethanol, which can account for the shift on the peak wavelength (Lakowicz, 2006). Hydrophobicity was calculated as the slope of the line between relative fluorescence at $2.0 \times$ $10^{-9} M$ and $4.0 \times 10^{-9} M$ ANS. Higher ANS concentrations $\left(>4.0 \times 10^{-9} M\right)$ resulted in saturation of the fluorescence intensity readings for some of the samples and these readings were not used for hydrophobicity calculations.

\section{RESULTS AND DISCUSSION}

\section{Absorbance}

The pasteurized skim milk samples with higher concentrations of ethanol (40 to 60\%) showed a temperature-dependent decrease in absorbance values, as shown in Figure 1. The samples containing $<30 \%$ ethanol showed little change in their absorbance values regardless of temperature. The observations were consistent with previous reports using nuclear magnetic resonance, confocal laser microscopy, and transmission spectroscopy to confirm the dissociation of the casein micelle mediated by heat and ethanol (O'Connell et al., 2001b, 2003).

When the temperature was $>45^{\circ} \mathrm{C}$, the samples containing $50 \%$ ethanol became translucent, which was consistent with previous reports (Dunkerley et al., 1993; Zadow, 1993) that reported changes in L-values (lightness) for milk and ethanol mixes. The decrease in the absorbance values confirms the dissociation of the milk casein micelles when heated in the presence of ethanol.

\section{Intrinsic Fluorescence}

Because of the presence of natural fluorophores, the $\mathrm{I}_{\text {If }}$ of raw skim milk, pasteurized skim milk, and WPI samples before the addition of ANS was measured $\left(\lambda_{\mathrm{EX}}\right.$ $\left.=390 \mathrm{~nm}, \lambda_{\mathrm{EM}}=450 \mathrm{~nm}\right)$. The properties of these natural fluorophores are sensitive to environmental 
conditions and changes in the 3-dimensional structure of the proteins (Kulmyrzaev et al., 2005). Both skim milk samples showed intrinsic fluorescence at the excitation wavelength used in the experiment, as seen in Figure 2. The intrinsic fluorescence was affected by the temperature and ethanol concentration in samples containing $>30 \%$ ethanol. In samples containing $<30 \%$ ethanol, there was no effect on the intrinsic fluorescence because of changes in temperature between 20 and $60^{\circ} \mathrm{C}$. These results were consistent with reports by Zadow (1993) showing that little dissociation of micelles occurred with ethanol concentrations $\leq 30 \%$, even at high temperature. In samples containing $>40 \%$ ethanol, the increase in temperature from 20 to $60^{\circ} \mathrm{C}$ resulted in a reduction in intrinsic fluorescence. To confirm that the fluorescence could be attributed to interactions within the casein micelles, we recorded the fluorescence intensity of samples containing only WPI. As seen in Figure 2, the pasteurized and raw skim milk samples showed very similar fluorescence patterns, and the WPI samples showed very little intrinsic fluorescence intensity to the point that it was considered negligible. The effect of heating on the intrinsic fluorescence of raw milk has been reported previously by Kulmyrzaev et al. (2005). By using front-face fluorescence to measure the effects of heat treatments on raw milk, Kulmyrzaev et al. (2005) observed that the intrinsic fluorescence of raw milk remained constant until a more aggressive treatment, $72^{\circ} \mathrm{C}$ for $30 \mathrm{~min}$, resulted in a slight decrease in the emission maximum. In our experiments using lower temperatures, both the raw and pasteurized skim milk samples showed intrinsic fluorescence patterns consistent with these reports. The intrinsic fluorescence of milk remained constant when no ethanol was added, but showed a similar reduction pattern when milk was heated in the presence of ethanol. Therefore, when milk is heated in the presence of ethanol, even at lower temperatures, it shows the same behavior as with a more aggressive higher temperature treatment. The observed intrinsic fluorescence behavior showed a correlation with the absorbance values of $\mathrm{r}=0.66(P$ $<0.0001)$ for raw skim milk and $\mathrm{r}=0.92(P<0.0001)$ for pasteurized skim milk.

\section{Extrinsic Fluorescence}

The extrinsic fluorescence intensity of the raw skim milk and pasteurized skim milk samples produced with the addition of ANS at a concentration of $2 \times 10^{-9} \mathrm{M}$ $\left(\mathrm{E}_{\text {If (ANS) }}\right)$ can be seen in Figure 3. For all the samples, the $\mathrm{E}_{\text {If (ANS) }}$ values decreased as the temperature and ethanol concentration increased. The largest decrease was observed in the raw and pasteurized skim milk samples containing $>50 \%$ ethanol. As seen in Figure

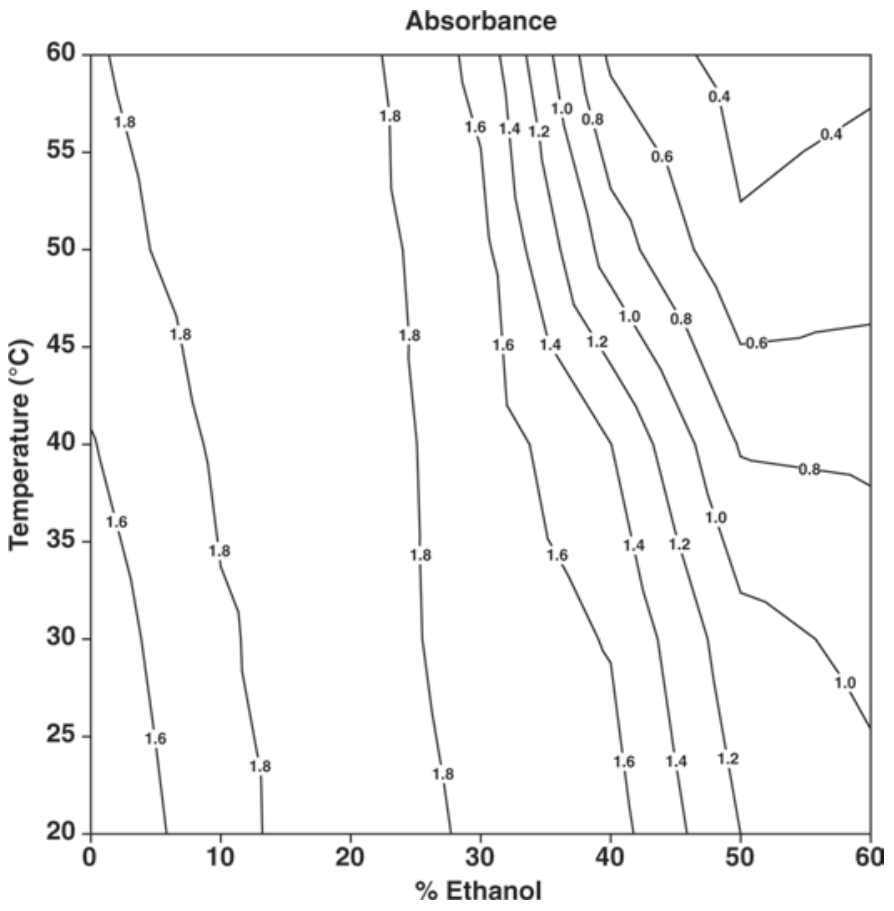

Figure 1. Absorbance of pasteurized skim milk (10\% vol/vol) solution measured at $500 \mathrm{~nm}$.

2 , the WPI samples showed very little native intrinsic fluorescence. However, as seen in Figure 3, the addition of ANS caused an increase in fluorescence intensity for samples with a higher ethanol concentration, regardless of the temperature. This influenced the $\mathrm{E}_{\text {If (ANS) }}$ for the raw and pasteurized skim milk samples because of some of the fluorescence in the samples being produced by the whey proteins. The WPI used in our study is considered a "native" isolate (Woodward et al., 2004) because low-heat spray drying has a minimal denaturing effect; therefore, we only adjusted the $\mathrm{E}_{\mathrm{If}(\mathrm{ANS})}$ for the raw skim milk sample. This adjusted $\mathrm{E}_{\text {If (ANS) }}$ can be seen in Figure 4. Although the unadjusted fluorescence values for the raw skim milk sample had a weak moderate correlation with the absorbance results $(\mathrm{r}=$ $0.58, P<0.001$ ), the adjusted $\mathrm{E}_{\mathrm{If}}$ (ANS) values had a stronger correlation values $(\mathrm{r}=0.71, P<0.001)$ with the absorbance. The $\mathrm{E}_{\mathrm{If}}$ (ANS) values for the pasteurized milk sample showed a strong correlation with the absorbance results $(\mathrm{r}=0.82, P<0.001)$. This further indicated that the decrease in $\mathrm{I}_{\mathrm{If}}$ for the samples was related to the dissociation of the milk casein micelle.

Two possible explanations for the decrease in fluorescence values are ethanol-induced changes to the globular structure of the whey proteins and competitive inhibition of ANS by ethanol. The effect of ethanol on the structure of $\beta$-lactoglobulin has been reported by Mousavi et al. (2008). In their study, conducted at room temperature, ethanol had no effect on the intrinsic fluo- 

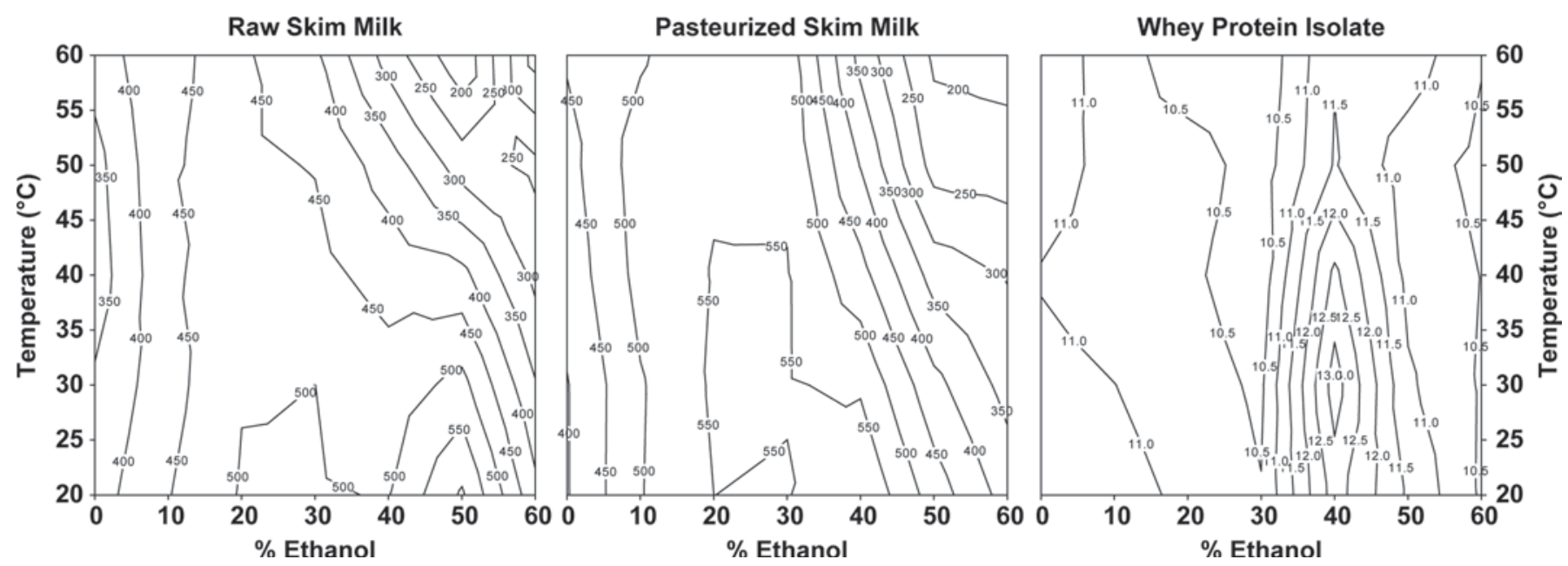

Figure 2. Intrinsic fluorescence intensity (excitation wavelength $\lambda_{\mathrm{EX}}=390 \mathrm{~nm}$, emission wavelength $\lambda_{\mathrm{EM}}=450 \mathrm{~nm}$ ) of raw skim milk, pasteurized skim milk, and whey protein isolate.

rescence of the protein. However, it did have an effect on the retinol binding ability of the protein. Mousavi et al. (2008) found no binding at ethanol concentrations $>40 \%$ ( vol/vol), which was attributed either to radical structural refolding of the protein or to competition by ethanol. In our experiments, the fluorescence of the whey proteins increased as the ethanol concentration of the samples increased, but showed little temperature effect. Ethanol showed an inhibitory effect on the binding of ANS to the casein proteins, resulting in lower extrinsic fluorescence values.

The extrinsic fluorescence results are consistent with prior reports of the behavior of ANS during changes to the surface hydrophobicity of milk proteins during heat treatments (Bonomi et al., 1988; Bonomi and Iametti, 1991; Risso et al., 2008). In our original hypothesis, after the micelles disassociate, the amount of hydrophobic sites available for ANS interaction should increase, resulting in an increase in fluorescence intensity. However, the opposite was observed. The change in intensity between intrinsic fluorescence and the fluorescence due to ANS interaction decreased as the temperature and ethanol concentration of the sample increased. This indicates that during the dissociation of the casein micelle, the $\mathrm{I}_{\mathrm{If}}$ of milk proteins was reduced. This reduction was also observed when more ANS was added to the samples, which indicates that ethanol has competitive inhibition with ANS. As the temperature
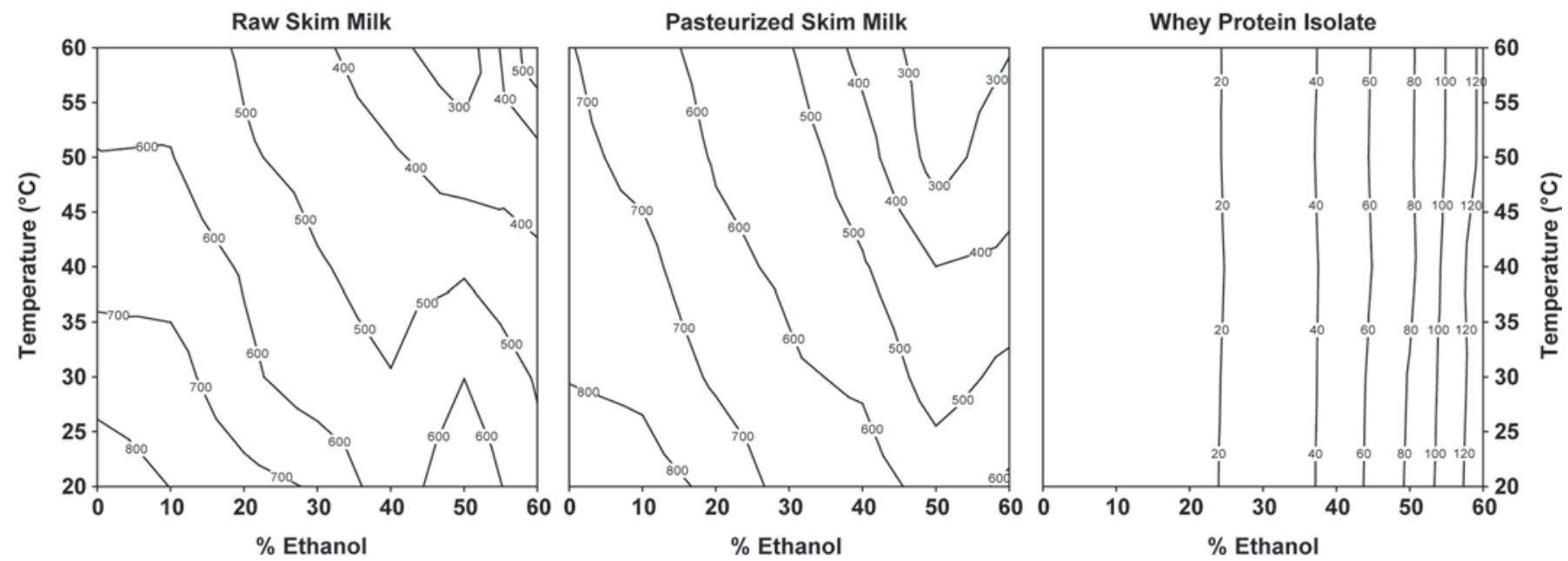

Figure 3. Extrinsic fluorescence intensity (excitation wavelength $\lambda_{\mathrm{EX}}=390 \mathrm{~nm}$, emission wavelength $\lambda_{\mathrm{EM}}=450 \mathrm{~nm}$ ) of raw skim milk, pasteurized skim milk, and whey protein isolates with the addition of 1 -anilinonaphthalene-8-sulfonic acid (ANS) to achieve a concentration of $2 \times 10^{-9} M$. 


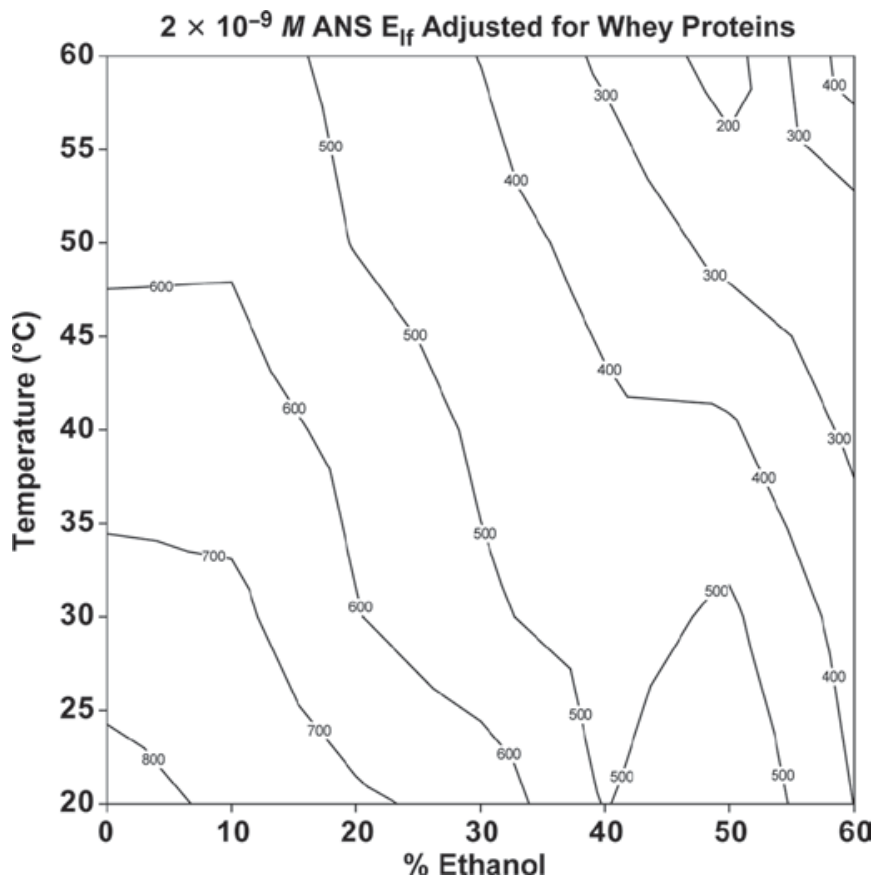

Figure 4. Adjusted extrinsic fluorescence intensity ( $\mathrm{E}_{\mathrm{If}(\mathrm{ANS})}$; excitation wavelength $\lambda_{\mathrm{EX}}=390 \mathrm{~nm}$, emission wavelength $\left.\lambda_{\mathrm{EM}}=450 \mathrm{~nm}\right)$ of casein and $2 \times 10^{-9} M$ 1-anilinonaphthalene-8-sulfonic acid (ANS) obtained by removing the fluorescence intensity of whey protein isolates with $2 \times 10^{-9} M$ ANS concentration from the fluorescence intensity of a raw skim milk $+2 \times 10^{-9} M$ ANS concentration.

increases, ethanol interferes with a greater number of hydrophobic zones and makes then unavailable for ANS interaction.

Figure 5 shows the change in the extrinsic fluorescence intensity of casein due to the addition of ANS $\left(\Delta \mathrm{E}_{\text {If (ANS) }}\right.$ of casein). In samples in which the ethanol concentration was $<30 \%$, the increase of temperature resulted in a slight reduction on the $\Delta \mathrm{E}_{\text {If (ANS) }}$ of casein values. In samples in which the ethanol concentration was $>30 \%$, the addition of ANS had little effect on the $\Delta$ casein $\mathrm{I}_{\text {If (ANS) }}$ values. Hence, the addition of ANS had no effect on the $\mathrm{I}_{\text {If }}$ of the caseins, which indicates that there were no available hydrophobic sites for ANS to interact with. Therefore, ethanol reduces the functional hydrophobicity of the casein micelles, because it essentially blocks the hydrophobic sites from being able to interact with ANS.

The competitive inhibition of ANS binding to hydrophobic sites by ethanol has been proposed in the past (Avdulov et al., 1996). The samples with and without ANS showed a decrease of $\mathrm{I}_{\mathrm{If}}$ as the temperature increased. This decrease of intensity is an indicator that the hydrophobicity of the proteins also decreases. The decrease in the hydrophobicity of the proteins can be attributed to a combination of the following factors:
A

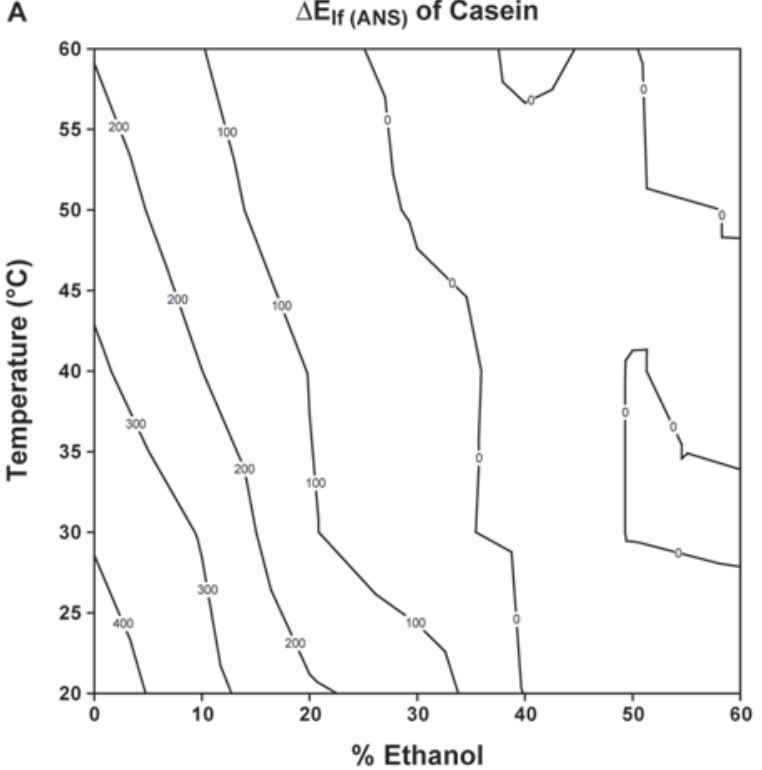

B

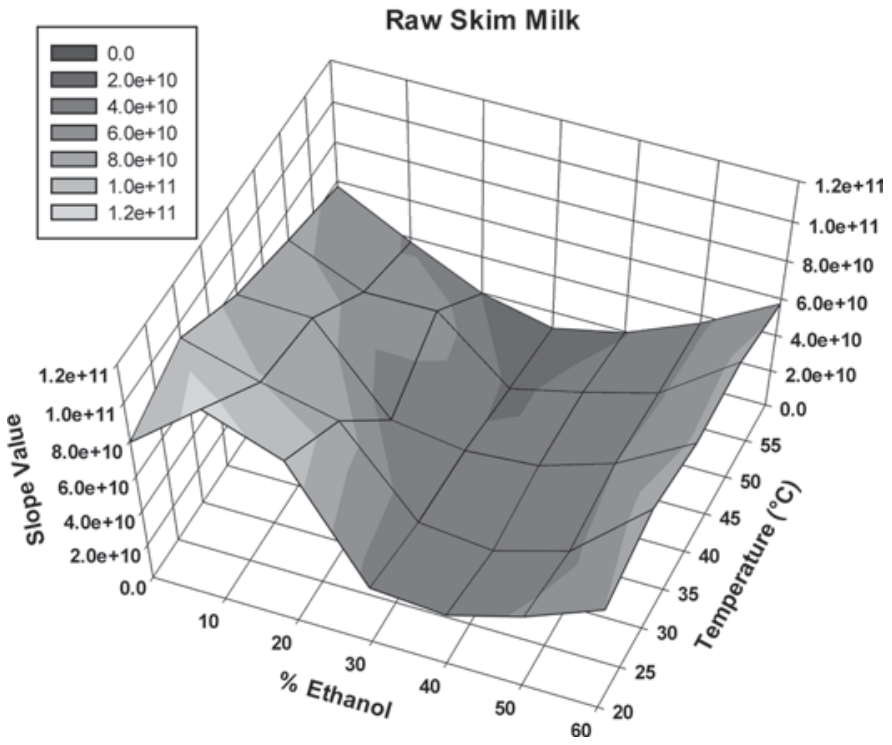

Figure 5. Change in the extrinsic fluorescence intensity $\left(\Delta \mathrm{E}_{\mathrm{If}(\mathrm{ANS})}\right)$ of casein due to the addition of 1-anilinonaphthalene-8-sulfonic acid (ANS) obtained by removing the fluorescence intensity of the whey proteins and the intrinsic fluorescence of raw skim milk.

whey protein restructuring and competition by ethanol for the diminishing number of hydrophobic sites.

\section{Hydrophobicity}

The calculated values for the slope of the line of the raw and pasteurized skim milk samples can be seen in Figure 6. This illustrates the effect that the increase in temperature has on the functional hydrophobicity of the milk casein micelles. At lower temperatures, the changes are limited to samples containing from 30 to 


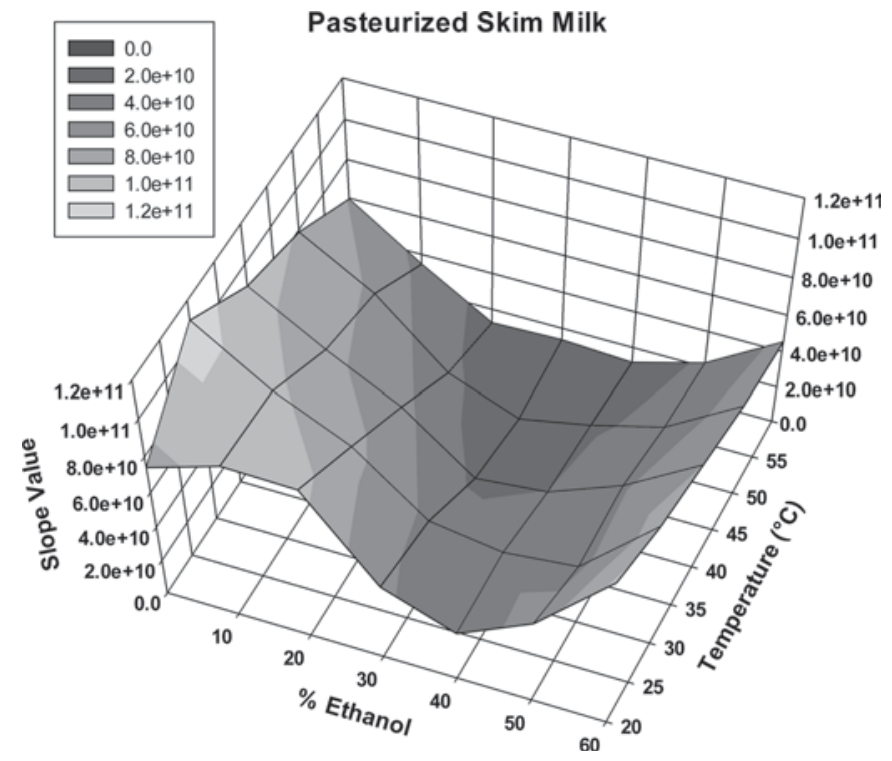

Figure 6. Differences in the value of the slope of the line calculated from the fluorescence intensity values of the samples when the concentration of 1-anilinonaphthalene-8-sulfonic acid (ANS) was increased from $2 \times 10^{-9} M$ to $4 \times 10^{-9}$ ANS.

$50 \%$ ethanol: ANS is able to compete with the ethanol and produce fluorescence. However, as the temperature increases, the range in which the hydrophobicity is lowered increases to include all samples that contain at least $20 \%$ ethanol. A higher concentration of ANS would be needed to cause a change in fluorescence.

Therefore, temperature and ethanol have a synergistic inhibitory effect on the interaction of ANS with the protein hydrophobic sites, which results in a lowering of the protein's functional hydrophobicity. However, our results indicate that ethanol exhibits competitive inhibition that can reduce the availability of hydrophobic sites. Further research concerning the nature of this inhibition may facilitate the use of dissociated casein micelles as transport vehicles.

\section{CONCLUSIONS}

By using ANS as a hydrophobic probe for fluorescence spectroscopy, we were able to track the changes in the functional hydrophobicity of casein proteins when milk casein micelles underwent ethanol-induced dissociation. We expected that the combined effect of ethanol and high temperature would expose functional hydrophobic sites of interaction within the individual casein proteins. This, in turn, would open the door to novel applications for the casein micelle such as the binding and stabilization of hydrophobic flavors in fluid foods. However, our results show that high ethanol content $(>30 \%)$ coupled with high temperature $\left(>40^{\circ} \mathrm{C}\right)$ reduce ANS fluorescence in dissociated casein micelles, suggesting a competitive inhibition of available hydrophobic sites for interaction. The nature and mechanism of this inhibition is still unknown.

\section{REFERENCES}

Alizadeh-Pasdar, N., and E. C. Y. Li-Chan. 2000. Comparison of protein surface hydrophobicity measured at various $\mathrm{pH}$ values using three different fluorescent probes. J. Agric. Food Chem. 48:328-334.

Altuner, E. M., H. Alpas, Y. K. Erdem, and F. Bozoglu. 2006. Effect of high hydrostatic pressure on physicochemical and biochemical properties of milk. Eur. Food Res. Technol. 222:392-396.

Avdulov, N. A., S. V. Chochina, V. A. Daragan, F. Schroeder, K. H. Mayo, and W. G. Wood. 1996. Direct binding of ethanol to bovine serum albumin: A fluorescent and C-13 NMR multiplet relaxation study. Biochemistry 35:340-347.

Bonomi, F., and S. Iametti. 1991. Real-time monitoring of the surface hydrophobicity changes associated with isothermal treatment of milk and milk protein-fractions. Milchwissenschaft 46:71-74.

Bonomi, F., S. Iametti, E. Pagliarini, and C. Peri. 1988. A spectrofluorometric approach to the estimation of the surface hydrophobicity modifications milk-proteins upon thermaltreatment. Milchwissenschaft 43:281-285.

Dunkerley, J. A., N. C. Ganguli, and J. G. Zadow. 1993. Reversible changes in the color of skim milk on heating suggest rapid reversible heat-induced changes in casein micelle size. Aust. J. Dairy Technol. 48:66-70.

Gatti, C. A., P. H. Risso, and M. S. Pires. 1995. Spectrofluorometric study on surface hydrophobicity of bovine casein micelles in suspension and during enzymatic coagulation. J. Agric. Food Chem. 43:2339-2344.

Hayakawa, S., and S. Nakai. 1985. Relationships of hydrophobicity and net charge to the solubility of milk and soy proteins. J. Food Sci. 50:486-491.

Kato, A., and S. Nakai. 1980. Hydrophobicity determined by a fluorescence probe method and its correlation with surfaceproperties of proteins. Biochim. Biophys. Acta 624:13-20.

Kulmyrzaev, A. A., D. Levieux, and E. Dufour. 2005. Front-face fluorescence spectroscopy allows the characterization of mild heat treatments applied to milk. Relations with the denaturation of milk proteins. J. Agric. Food Chem. 53:502-507.

Lakowicz, J. R. 2006. Principles of Fluorescence Spectroscopy. Springer, New York, NY.

Mousavi, S. H. A., J. M. Chobert, A. K. Bordbar, and T. Haertlé. 2008. Ethanol effect on the structure of beta-lactoglobulin B and its ligand binding. J. Agric. Food Chem. 56:8680-8684.

Nakai, S., and E. Li-Chan. 1998. Hydrophobic interactions in food systems. CRC Press Inc., Boca Raton, FL.

O'Connell, J. E., A. L. Kelly, M. A. E. Auty, P. F. Fox, and K. G. de Kruif. 2001a. Ethanol-dependent heat-induced dissociation of casein micelles. J. Agric. Food Chem. 49:4420-4423.

O'Connell, J. E., A. L. Kelly, P. F. Fox, and K. G. de Kruif. 2001b. Mechanism for the ethanol-dependent heat-induced dissociation of casein micelles. J. Agric. Food Chem. 49:4424-4428.

O'Connell, J. E., S. Steinle, F. Reiter, M. A. E. Auty, A. L. Kelly, and P. F. Fox. 2003. Properties of casein micelles reformed from heated mixtures of milk and ethanol. Colloid Surf. A Physicochem. Eng. Asp. 213:265-273.

Risso, P. H., D. M. Borraccetti, C. Araujo, M. E. Hidalgo, and C. A. Gatti. 2008. Effect of temperature and $\mathrm{pH}$ on the aggregation and the surface hydrophobicity of bovine kappa-casein. Colloid Polym. Sci. 286:1369-1378.

Woodward, N. C., P. J. Wilde, A. R. Mackie, A. P. Gunning, P. A. Gunning, and V. J. Morris. 2004. Effect of processing on the displacement of whey proteins: Applying the orogenic model to a real system. J. Agric. Food Chem. 52:1287-1292.

Zadow, J. G. 1993. Alcohol-mediated temperature-induced reversible dissociation of the casein micelle in milk. Aust. J. Dairy Technol. 48:78-81. 